\title{
Nécrolyse épidermique toxique type Steven-Johnson induite par l'association de métronidazole et de spiramycine pour le traitement d'une infection dentaire
}

\section{Metronidazole and Spiramycin Induced Toxic Epidermal Necrolysis in Dental Infection}

\author{
N. Guyomarc'h $\cdot$ B. Muller $\cdot$ X. Combes \\ Reçu le 8 septembre 2017; accepté le 24 janvier 2018 \\ (C) SFMU et Lavoisier SAS 2018
}

\section{Introduction}

Les nécrolyses épidermiques toxiques sont des réactions rares, potentiellement mortelles. L'incidence est d'un à trois cas par million d'habitants et par an en Europe [1,2]. La mortalité est estimée à $23 \%$ [2]. Le tableau évocateur comprend un décollement cutané, une altération de l'état général, une fièvre, avec une atteinte systématique des muqueuses. Une cause d'origine médicamenteuse est trouvée dans la majorité des cas. Les principaux médicaments responsables de ces syndromes sont les sulfamides antibactériens, l'allopurinol, les antiépileptiques (carbamazépine, phénytoïne, phénobarbital, lamotrigine) et les anti-inflammatoires non stéroïdiens (oxycam) [2-4]. Jusqu'à présent, le métronidazole et la spiramycine sont des molécules peu connues pour déclencher de telles réactions. Nous rapportons le cas d'une patiente de 53 ans prise en charge au service d'accueil des urgences pour un syndrome de Steven-Johnson secondaire à une antibiothérapie associant métronidazole et spiramycine.

\section{Observation}

Une patiente, âgée de 53 ans, s'est présentée chez son médecin traitant pour infection dentaire. La patiente n'avait pas d'antécédent connu. Elle présentait une allergie à la nitrofurantoïne sous forme d'urticaire. Une antibiothérapie par

N. Guyomarc'h $\cdot$ X. Combes

Service des urgences/SAMU/SMUR/EVASAN, CHU de Saint-Denis, université de La Réunion, Site Félix Guyon - SAMU - Allée des Topazes, 97400 Saint-Denis, La Réunion

B. Muller $(\square)$

Service des urgences/SAMU/SMUR/EVASAN, CHU de Saint-Denis, Site Félix Guyon - SAMU -

Allée des Topazes, 97400 Saint-Denis, La Réunion

e-mail : bagbagamuller@hotmail.com métronidazole et spiramycine a été débutée. Cinq jours après le début du traitement, la patiente a consulté en médecine de ville pour des lésions maculopapuleuses sur le visage et le décolleté. Un traitement par corticoïdes et antihistaminique a été initié et l'antibiothérapie a été arrêtée par le médecin. Cinq jours plus tard, devant l'aggravation des lésions, la patiente a consulté de nouveau son médecin traitant qui l'a adressée aux urgences.

À l'arrivée aux urgences, la patiente était apyrétique et en bon état général. Les paramètres vitaux comprenaient une tension artérielle à $130 / 78 \mathrm{mmHg}$, un pouls à $105 \mathrm{bpm}$, une température à $37,2{ }^{\circ} \mathrm{C}$, une saturation à $98 \%$ et une glycémie à $5,39 \mathrm{mmol} / \mathrm{l}$. L'examen cutané retrouvait un érythème avec décollement cutané au niveau du visage, du cou, de la partie supérieure du thorax et des deux membres supérieurs (Fig. 1). La surface cutanée atteinte a été évaluée à $9 \%$. Les lésions étaient légèrement prurigineuses. Une atteinte de la muqueuse buccale a été retrouvée, avec une bulle sur le voile du palais, une desquamation des lèvres et un décollement de la partie interne de la lèvre inférieure. Il n'y avait pas de signe de surinfection des lésions. L'examen respiratoire était sans anomalie.

Le bilan biologique était sans anomalie. Le taux de polynucléaires éosinophiles était à $0,29 \mathrm{G} / 1$. Le traitement a compris une réhydratation intraveineuse, une antalgie par paracétamol et des soins locaux. Aucune antibioprophylaxie n'a été débutée. Ni corticothérapie ni traitement par immunoglobine n'ont été débutés après avis spécialisé. La patiente a été hospitalisée par la suite en service de dermatologie, devant la forte suspicion de nécrolyse épidermique. Le diagnostic retenu était un syndrome de Steven-Johnson. La biopsie cutanée a retrouvé une bulle intraépidermique secondaire et une nécrose kératinocytaire. Le derme superficiel renfermait un discret infiltrat inflammatoire mononucléé, de topographie périvasculaire avec de rares éosinophiles. L'immunofluorescence directe était négative. Le compte-rendu anatomopathologique était compatible 


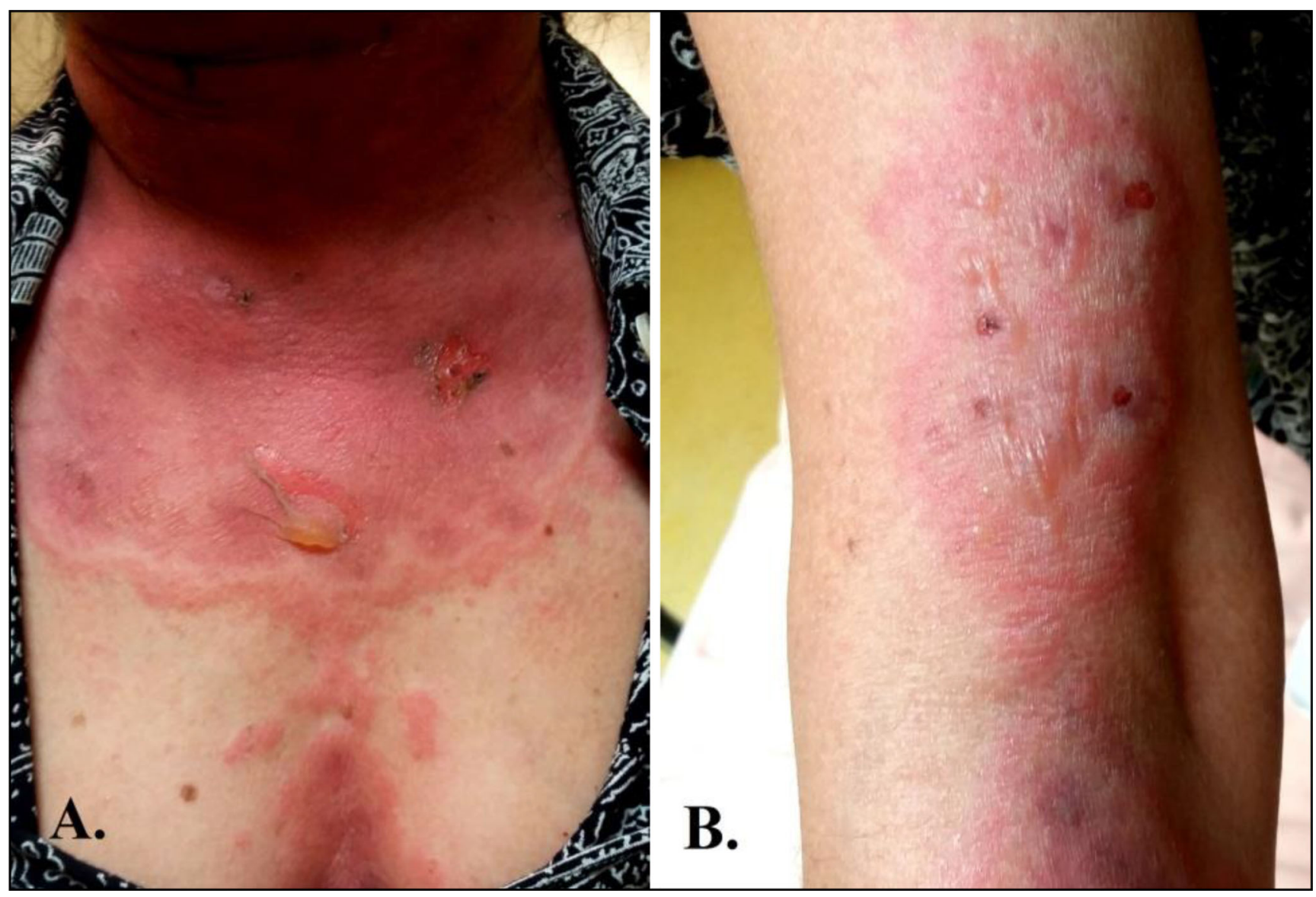

Fig. 1 A. Partie supérieure du thorax avec un érythème et décollement cutané. B. Face antérieure du membre supérieur droit avec œedème cutané et aspect « bulleux ». Reproduit avec l'autorisation de la patiente

avec une toxidermie. Les recherches d'anticorps antidesmogléine 1 et 3 et d'anticorps antisubstance intercellulaire se sont révélées négatives, éliminant les diagnostics différentiels de dermatoses bulleuses auto-immunes.

L'évolution a été favorable en quelques jours. Une déclaration à la pharmacovigilance de Bordeaux a été réalisée. Un certificat de contre-indication au métronidazole et à la spiramycine a été remis à la patiente. La consultation de contrôle, un mois après la sortie d'hospitalisation de la patiente, a permis de constater une cicatrisation complète des lésions cutanées et muqueuses.

\section{Discussion}

Les nécrolyses épidermiques sont liées à des réactions immunitaires médiées par les lymphocytes T CD8. Ils agissent par la reconnaissance d'un complexe médicament/peptide/human leukocyt antigen (HLA) qui active les lymphocytes et induit la libération de molécules cytotoxiques responsables de la mort des kératinocytes et plus généralement des cellules épithéliales [5]. Le risque est plus accru chez la femme et après 40 ans
[1]. Il existe une prédisposition génétique liée à des groupes HLA favorisants expliquant une incidence plus élevée à Taïwan. L'incidence est par ailleurs 10 à 100 fois plus importante dans l'infection par le virus de l'immunodéficience humaine (VIH) et durant le stade de syndrome d'immunodéficience acquise (sida) [1,2].

L'agent causal est souvent difficile à déterminer avec certitude. Dans ce cas, la patiente n'avait reçu aucun autre traitement en dehors du paracétamol. Il existe des outils stratifiant la probabilité d'implication d'un médicament. L'imputabilité intrinsèque d'un médicament comprend des critères chronologiques et sémiologiques. Ces critères concernent, d'une part, le délai d'apparition des symptômes et l'évolution des symptômes à l'arrêt du médicament et à sa réintroduction, d'autre part, les éléments cliniques et paracliniques compatibles ainsi que l'absence de diagnostic différentiel. Le tableau d'imputabilité issu de Mokline et al. [6] a été utilisé pour évaluer le traitement incriminé. L'imputabilité du métronidazole et de la spiramycine associée a été définie comme « vraisemblable » dans la survenue du syndrome de StevenJohnson chez cette patiente. La chronologie était « plausible » et la sémiologie " vraisemblable ». Après contact avec 
l'ANSM (Agence nationale de sécurité du médicament et des produits de santé), la survenue de syndromes de Lyell et Steven-Johnson est bien spécifiée dans le résumé des caractéristiques du produit pour le métronidazole et pour la spiramycine, mais la fréquence est indéterminée. L'unité de pharmacovigilance de Bordeaux compte, en Europe, 57 cas de Steven-Johnson sous métronidazole, 6 cas sous spiramycine et seulement deux cas sous l'association des deux molécules. Par ailleurs, l'utilisation de l'algorithme ALDEN attribue une imputabilité « probable » au métronidazole et à la spiramycine avec un score de 4 (délai compatible +3 et molécules suspectées +1) [7].

Très peu d'études rapportent des cas de nécrolyses épidermiques suite à la prise de métronidazole ou de spiramycine. Quelques cas isolés seraient secondaires à la prise de macrolides, mais n'impliquant pas la spiramycine [8-10]. Le métronidazole est incriminé en association avec le mébendazole dans une étude taïwanaise et de façon isolée sur un cas en Inde dans un contexte d'infection dentaire également [11,12].

Devant les éléments cliniques et paracliniques présentés par la patiente, le diagnostic de syndrome de Steven-Johnson sur la prise de métronidazole et spiramycine semble le plus probable. Le délai habituel d'apparition des symptômes est respecté, soit entre 7 et 21 jours [4]. La présentation clinique de notre patiente était toutefois atypique, car il existait une atteinte cutanée et une atteinte des muqueuses, mais ni l'altération de l'état général ni la fièvre n'étaient présentes. Les diagnostics différentiels sont : le syndrome d'épidermolyse staphylococcique avec une atteinte de l'épiderme superficiel à la biopsie, sans atteinte des muqueuses ; les dermatoses bulleuses auto-immunes avec un dépôt d'immunoglobulines à la biopsie et d'autres toxidermies (DRESS syndrome et érythème pigmenté fixe). Dans ce cas, ces diagnostics n'étaient pas compatibles $[2,13]$. Le traitement par corticoïdes et immunoglobines reste controversé et non recommandé [14]. Le tableau clinique initial ne présentait pas de facteur de mauvais pronostic. Le score de SCORTEN prédit le pourcentage de décès selon différents critères (âge, fréquence cardiaque, pourcentage de surface décollée, urémie, bicarbonatémie, glycémie) [15]. La patiente avait un score à 1 , soit un risque de décès autour de 3,2\%.

En conclusion, devant la gravité de telles réactions, et malgré la rareté de cette pathologie, il est important de savoir évoquer le diagnostic de nécrolyse épidermique toxique aux urgences. Nous rapportons ici un cas rare de cette pathologie secondaire à l'utilisation d'une association de métronidazole et de spiramycine.

Liens d'intérêts : Les auteurs déclarent ne pas avoir de liens d'intérêts.

\section{Références}

1. Roujeau JC (2007) Syndromes de Lyell et de Stevens-Johnson. Rev Prat 57:1165-70

2. Bensaid B, Nicolas JF, Valeyrie-Allanore L, et al (2015) Hypersensibilité aux médicaments. Exanthèmes médicamenteux et toxidermie sévères. Signes généraux et muqueux imposent l'arrêt immédiat du médicament. Rev Prat 65:981-4

3. Roujeau JC, Kelly JP, Naldi L, et al (1995) Medication use and the risk of Stevens-Johnson syndrome or toxic epidermal necrolysis. N Engl J Med 333:1600-8

4. Thielen AM, Toutous-Trellu L, Desmeules J (2008) Les toxidermies médicamenteuses. Rev Med Suisse 4:1671-5

5. Simon M (2009) Activation des lymphocytes. Cours Pharmacie. http://www.cours-pharmacie.com/immunologie/activation-deslymphocytes.html (Dernier accès le 20 octobre 2017)

6. Mokline A, Rahmani I, Garsallah L, et al (2016) La nécrolyse épidermique toxique. Ann Burns Fire Disasters 29:37-40

7. Sassolas B, Haddad C, Mockenhaupt M, et al (2010) ALDEN, an algorithm for assessment of drug causality in Stevens-Johnson syndrome and toxic epidermal necrolysis: Comparison with case-control analysis. Clin Pharmacol Ther 88:61-8

8. Khladi N, Miras A, Gromb S (2005) Toxic epidermal necrolysis and clarithromycin. Can J Clin Pharmacol 12:e264-8

9. Masia M, Gutteriez F, Jimeno A (2002) Fulminant hepatitis and fatal toxic epidermal necrolysis (Lyell disease) coincident with clarithromycin administration in an alcoholic patient receiving disulfiram therapy. Arch Intern Med 162:474-6

10. Das S, Mondal S, Dey JK (2012) Roxithromycin-induced toxic epidermal necrolysis. Ther Drug Monit 34:359-62

11. Chen KT, Twu SC, Chang HJ, et al (2003) Outbreak of StevensJohnson syndrome/toxic epidermal necrolysis associated with mebendazole and metronidazole use among filipino laborers in Taiwan. Am J Public Health 93:489-92

12. Mazumdar G, Shome K (2014) Stevens-Johnson syndrome following use of metronidazole in a dental patient. Indian J Pharmacol 46:121-2

13. Galetto A (2013) Syndrome de Stevens-Johnson http://raft.g2hp. net/wp-content/blogs.dir/9/files/2014/09/Syndrome-de-stevensJohnson.pdf (Dernier accès le 20 octobre 2017)

14. Ye LP, Zhang C, Zhu QX (2016) The effect of intravenous immunoglobulin combined with corticosteroid on the progression of Stevens-Johnson syndrome and toxic epidermal necrolysis: a meta-analysis. PLoS One 11:e0167120

15. Bastuji-Garin S, Fonchard N, Bertocchi M, et al (2000) SCORTEN: a severity-of-illness score for toxic epidermal necrolysis. J Invest Dermatol 115:149-53 